\title{
How to Optimize the Use of Blood Cultures for the Diagnosis of Bloodstream Infections? A State-of-the Art
}

\author{
Brigitte Lamy ${ }^{1 * t}$, Sylvie Dargère ${ }^{2 \dagger}$, Maiken C. Arendrup ${ }^{3}$, Jean-Jacques Parienti ${ }^{4}$ and \\ Pierre Tattevin ${ }^{5}$
}

${ }^{1}$ Laboratoire de Bactériologie, CHU Montpellier, Montpellier, France, ${ }^{2}$ Service de Maladies Infectieuses, CHU Caen, Caen, France, ${ }^{3}$ Unit for Mycology, Department of Microbiology \& Infection Control, Statens Serum Institut, Copenhagen, Denmark, ${ }^{4}$ Unité de Biostatistiques et de Recherche Clinique, CHU Caen, Caen, France, ${ }^{5}$ Service de Maladies Infectieuses, CHU Rennes, Rennes, France

OPEN ACCESS

Edited by:

Gillbert Greub,

University of Lausanne, Switzerland

Reviewed by:

Max Maurin,

Assistance Publique-Hôpitaux de Marseille and Université Aix-Marseille II, France

Tamas Szakmany

Cardiff University, UK

*Correspondence:

Brigitte Lamy

brigitte_lamy@yahoo.fr

${ }^{\dagger}$ These authors have contributed equally to this work

Specialty section: This article was submitted to Infectious Diseases,

a section of the journal Frontiers in Microbiology

Received: 13 February 2016 Accepted: 26 April 2016 Published: 12 May 2016

Citation:

Lamy B, Dargère S, Arendrup MC, Parienti J-J and Tattevin P (2016) How to Optimize the Use of Blood Cultures for the Diagnosis of Bloodstream Infections? A State-of-the Art. Front. Microbiol. 7:697.

doi: 10.3389/fmicb.2016.00697
Bloodstream infection (BSI) is a major cause of death in developed countries and the detection of microorganisms is essential in managing patients. Despite major progress has been made to improve identification of microorganisms, blood culture $(\mathrm{BC})$ remains the gold standard and the first line tool for detecting BSIs. Consensus guidelines are available to ensure optimal BSI procedures, but BC practices often deviate from the recommendations. This review provides an update on clinical and technical issues related to blood collection and to BC performance, with a special focus on the blood sample strategy to optimize the sensitivity and specificity of BCs.

Keywords: bloodstream infection, blood culture, single sampling strategy, multi-sampling strategy, sensitivity, specificity, contamination

\section{INTRODUCTION}

Bloodstream infections (BSIs) represent a growing public health concern, with an estimated burden of 1,200,000 episodes of BSI each year in Europe, and 157,000 attributable deaths (Goto and Al Hasan, 2013). BSI ranks among the top seven causes of death in North America and Europe. The diagnosis of BSI relies on the documentation of pathogens in blood-either bacteremia, or fungemia-through blood cultures (BCs). Indeed, although the last decades have experienced dramatic achievements in the development of rapid diagnostic tests relying on innovative technologies, BCs remain the gold-standard not only for the diagnosis of BSI, but also for the identification of the responsible pathogen(s), and for the testing of their susceptibility to antiinfective agents. Since the mid-1970s, various original studies, systematic reviews, and guidelines to better define the principles and practices of BCs sampling and processing have been published. The available evidence suggests that the diagnostic yield of BCs is influenced by the collection of appropriate specimens, from selected patients with reasonable suspicion of BSI. We performed a systematic literature review on clinical and technical issues related to blood collection, as well as interpretation of BCs, in adult patients suspected of BSI. We focused on the impact of BC collection strategies on their performance for the diagnosis of BSI, as this has not been a major focus in most recent reviews (e.g., Kirn and Weinstein, 2013; Garcia et al., 2015). 


\section{ORDERING BLOOD CULTURES}

Published guidelines do not clearly state when BCs should be ordered (Baron et al., 2013). Blood cultures are commonly collected when patients have fever, chills, leukocytosis, septic shock, suspected endocarditis or prior to starting antimicrobial treatment in elderly or immunocompromised patients.

Physicians significantly overestimate the likelihood of BSI for their patients (Poses and Anthony, 1991). Indeed, in most settings, only 5 to $13 \%$ of BCs will turn out to be positive, and of those, 20-56\% represent contaminants (Bates et al., 1990; Salluzzo and Reilly, 1991; Little et al., 1997; Dargère et al., 2014). Despite progress in skin antisepsis since 25 years led to lower risk of contamination (e.g., $0.5-1 \%$, Garcia et al., 2015), rates of contamination as high as $2.1-6 \%$ are still commonly reported, and the $20-56 \%$ proportion of contaminants holds true today (e.g., Zwang and Albert, 2006; Gander et al., 2009; Roth et al., 2010; Dargère et al., 2014).

Many models for predicting bacteremia have been developed but not all were validated and, when they were, the validation processes were highly heterogeneous (Bates et al., 1990; Shapiro et al., 2008; Coburn et al., 2012). Eliakim-Raz et al. (2015) identified studies that underwent validation on prediction of bacteremia and that were able to define groups with low $(<3 \%)$ or high $(>30 \%)$ probabilities of bacteremia in adults. They demonstrated that few studies have been prospectively validated, populations and parameters included were heterogeneous and none of these models were implemented in clinical practice (Eliakim-Raz et al., 2015). The additional workload required to enter data may explain the reluctance to use these models. Multicenter clinical trials would be necessary to better define the added value of models to guide $\mathrm{BC}$ ordering strategies in clinical practice.

\section{TIMING OF BC SPECIMEN COLLECTION}

Thus far, the most appropriate timing of $\mathrm{BC}$ collection has been poorly evaluated through clinical studies. Most guidelines state that blood specimens should be collected in the absence of antimicrobials, at or around the time of fever spikes, and a 30-60 min interval between samples has been arbitrarily recommended (Weinstein, 1996). However, in a seven-center study that evaluated the timing of $\mathrm{BC}$ collection in relation to body temperature in 1436 patients with BSI, Riedel et al. (2008) could not identify any optimal timing for BC collection. Indeed, the likelihood of documenting BSIs was not significantly enhanced by collecting blood during temperature spikes. Yields were similar over a 24-h period before and after temperature spikes (Riedel et al., 2008). Consistent with these results, Li et al. (1994) found no difference in BCs yield whether samples were collected within a 24 -h period, either simultaneously or serially (Li et al., 1994).

\section{BLOOD CULTURE COLLECTION}

\section{Skin Preparation}

Meticulous skin antisepsis at the time of blood collection is paramount to reduce the risk of $\mathrm{BC}$ contamination, so that the rate of contaminants would remain below the threshold of $3 \%$ of all BCs sampled (Garcia et al., 2015). In a systematic review, Malani et al. (2007) found that: (i) alcoholic iodine tincture is more effective than aqueous povidone-iodine (PVI) to reduce the risk of $\mathrm{BC}$ contamination; (ii) alcoholic chlorhexidine gluconate $(\mathrm{CHG})$ is more effective than aqueous PVI; (iii) alcoholic antiseptics are more effective than aqueous products, and (iv) that alcohol alone is not inferior to any iodine products (Malani et al., 2007). In a more recent meta-analysis of 6 clinical trials, alcoholic chlorhexidine was found to be more effective than non-alcoholic PVI, while alcoholic solutions were more effective than non-alcoholic solutions. This review found no significant difference between chlorhexidine and iodine products for skin antisepsis before blood collection (Caldeira et al., 2011). The Clinical and Laboratory Standards Institute (CLSI) guidelines for BCs collection favor the use of CHG for infants $>2$ months of age, children and adults (Wilson et al., 2007); and the Food and Drug administration recommended to use it with care in premature infants or infants under 2 months of age. These products may cause irritation or chemical burns (Schiffenbauer, 2012).

A recent randomized crossover trial comparing the effectiveness of 3 skin antiseptic interventions-10\% aqueous PVI solution, 2\% iodine tincture (IT), and 2\% CHG in $70 \%$ isopropyl alcohol-before BCs sampling, found that the choice of antiseptic agent does not impact contamination rates when $\mathrm{BCs}$ are sampled by a dedicated phlebotomy team (Washer et al., 2013). A systematic review and meta-analysis concluded that the implementation of dedicated phlebotomy teams reduce the rate of BCs, while no evidence support the use of prepackaged prep kits (Snyder et al., 2012). In addition, it has been demonstrated that BCs contamination rates are significantly lower when an antiseptic agent is applied on BC bottle tops before sampling (Schifman et al., 1998). European and French guidelines recommend the use of an alcoholic solution for antisepsis before BC sampling (Lamy and Seifert, 2012; Accoceberry et al., 2015a).

For an extensive review on skin antisepsis before BC collection and prepackaged kits performance, see Garcia et al. (2015).

\section{Sampling Site}

Several studies concluded that peripheral venipuncture is the method of choice for BC collection, as compared with sampling through intravenous catheter, with rates of contamination ranging from 1.2 to $7.3 \%$ when samples are obtained from venipuncture compared with 3.4 to $13 \%$ when blood is drawn through catheter (Dawson, 2014; Garcia et al., 2015). Indeed, both colonization of the catheter, and breakdowns in sterile procedure increase the risk of BCs contamination when BCs samples are drawn through these devices (Bates et al., 1991). A multi-interventional study that included education of healthcare workers to avoid the sampling of BCs from central intravenous lines, documented simultaneous decreases in (i) the proportion of BCs obtained from central lines (from 10.9 to $0.4 \%$ ); and (ii) BCs contamination rates (from 1.6 to $0.5 \%$; Boyce et al., 2013). Although studies have shown that contamination rates are lower for BCs drawn from newly inserted catheter using a sterile technique protocol (Levin et al., 2013), BCs are usually even less contaminated when samples are drawn by peripheral venipuncture (Snyder et al., 2012). 
One exception must be outlined: for the diagnosis of central line associated BSI (CLABSI), most current guidelines recommend simultaneous sampling of $\mathrm{BC}$ drawn from the suspected catheter, and through a venipuncture, to be able to estimate the differential time to positive BC (Baron et al., 2013; Dellinger et al., 2013). Indeed, if BC drawn from the central line grows at least $2 \mathrm{~h}$ earlier than $\mathrm{BC}$ drawn from venipuncture, the central line is most likely the source of BSI (Kirn and Weinstein, 2013). However, improper collection of BCs is associated with potential over-reporting of CLABSI (Kaasch et al., 2014; Garcia et al., 2015).

Despite concordant recommendations, the techniques for collection of BCs vary across countries in routine practice. A study performed in intensive care units across 4 European countries found that the preferred means for BCs collection were peripheral venipuncture in Germany and Italy (42 and $76 \%$ via peripheral venipuncture, respectively, vs. 8 and $0 \%$ via intravenous catheter, while 50 and $24 \%$ reported no preference), while sampling through intravenous catheter was more common in France and the UK (respectively, 33 and 23\% via intravenous catheter, vs. 20 and $23 \%$ via peripheral venipuncture, while 47 and $54 \%$ reported no preference; Schmitz et al., 2013).

\section{Impact of Volume Sampled on BCs Yield}

Data available could be summarized as follows: "The higher the blood volume cultured, the higher the yield." Indeed, adequate volume sampling is the most important parameter for the detection of bloodstream microorganisms because bacterial or fungal density in blood is very low in most patients with BSI. Basically, the likelihood of detecting a BSI depends on the bacterial or fungal concentration, and on the volume collected. Using theoretical models, Arpi et al. (1989) estimated the average bacterial concentration in patients with bacterial BSI at 0.25 colony-forming unit (CFU) per milliliter, and Jonsson et al. (1993) demonstrated that the bacterial concentration was less than $0.04 \mathrm{CFU} / \mathrm{mL}$ in $29 \%$ of Escherichia coli BSI and $18 \%$ of Staphylococcus aureus BSI (Arpi et al., 1989; Jonsson et al., 1993). The sensitivity of $\mathrm{BC}$ was estimated to be $95 \%$ when $3 \mathrm{CFU}$ are sampled, which implies that at least $30 \mathrm{~mL}$ of blood are incubated (Jonsson et al., 1993). The results of this model are in full agreement with Washington's empirical data obtained 18 years earlier, showing that a total volume of at least $30 \mathrm{ml}$ of blood is required for detecting 99\% of BSI (Washington, 1975). There are very few clinical studies directly evaluating the bacterial concentration in BSI, because this measurement requires the use of quantitative BC techniques, which are particularly laborintensive, and not routinely used. These studies are consequently either originating from the early years of BCs, decades ago (with potential limitation due to the quality of the culture media used), or more recent, based on the lysis-centrifugation technique, but limited to a specific type of BSI (Tables 1, 2). For instance, Wain et al. (1998) showed that $53 \%$ of 349 patients with enteric fever had a Salmonella enterica serovar Typhi concentration between 0.01 and $1 \mathrm{CFU} / \mathrm{mL}$ of blood (Wain et al., 1998). In Reynes's study, $54.3 \%$ of 1026 patients with BSI (any type) had a bacterial density between 0.1 and 2 CFU/ mL of blood (Reynes, 1947). Similar data have been observed with Candida BSI (Table 2), and in Pfeiffer et al.'s study, 53\% of 152 patients had a fungal density of less than $1 \mathrm{CFU} / \mathrm{mL}$ (Pfeiffer et al., 2011). Overall, data from modeling, as well as clinical studies are remarkably concordant: $50 \%$ of BSI episodes are associated with a bacterial concentration in the range of 0.01-1 CFU/mL (Tables 1, 2).

Comparing the yield of standard-volume BC (mean, $8.7 \mathrm{~mL}$ ), and low-volume BC (mean, $2.7 \mathrm{~mL}$ ), a study from the Wisconsin Hospital and Clinics demonstrated that the sensitivity of BCs for the diagnosis of BSI was 92\% with standard-volume, and $69 \%$ with low-volume (difference of 23\%, [95\% CI, 9-37\%]; $P<0$, 001; Mermel and Maki, 1993). Examining 7783 BCs, including 624 classified as true positive BCs, Li et al. (1994) demonstrated that increasing the volume of blood cultured from 20 to $40 \mathrm{~mL}$ increased the yield by $19 \%$, with an additional gain of $10 \%$ when the incubated volume was increased from 40 to $60 \mathrm{~mL}$ (Li et al., 1994). Since then, several studies have confirmed that the volume of blood cultured is the key parameter of BC yield (Cockerill et al., 2004; Patel et al., 2011). The most recent studies showed that sampling blood volumes of 20,40 , and $60 \mathrm{~mL}$ was associated with sensitivities of $65.0-75.7 \%, 80.4-89.2 \%$, and $95.7-97.7 \%$, respectively (Cockerill et al., 2004; Bouza et al., 2007; Lee et al., 2007; Patel et al., 2011).

Of note, since Washington's seminal works (Washington, 1975), the recommended volume of blood to be cultured gradually increased over decades. This increase resulted both from the need to develop (and accept) the concept of culturing larger volumes of blood, and from the study design on which data relied on: in the absence of perfect gold standard for detecting BSI, the $100 \%$ of BSI detected in each study is bounded by the maximum volume of blood cultured, with an inherent risk to under-detect BSI in case of lower volumes. Finally, since the 1970's, the changes in BSI epidemiology that have been reported may have been in part impacted by these changes in sampling techniques, and culture media system: as most BSI are associated with a low bacterial or fungal density, improvement in BCs sensitivity most likely increased the incidence of documented BSI, and may also have impacted on their patterns.

\section{Current Guidelines}

As they are based on similar literature data, guidelines from scientific societies are in agreement on most issues. The UK Standards for Microbiology Investigations recommend 4 bottles (2 sets), corresponding to $20-30 \mathrm{~mL}$ per set (Public Health England, 2014). The French Society of Microbiology recommends that, in patients suspected of BSI, 4-6 bottles ( 2 to 3 sets) of blood should be cultured, with adequate volume for each bottle. A 6 bottles-procedure is necessary whenever the optimal filling of all the bottles is not ensured (Accoceberry et al., 2015a). The European guidelines also recommend the culture of 4-6 bottles adequately filled (Lamy and Seifert, 2012). The American College of Critical Care Medicine (ACCM) and Infectious Diseases Society of America (IDSA) Guidelines recommend that new fever in critically ill adult patients must be investigated by drawing of 3-4 BC sets with appropriate volume within the $24 \mathrm{~h}$ of fever onset (O'Grady et al., 2008). Guidelines from the American Society of Microbiology (ASM) 
TABLE 1 | Bacterial concentration in blood from adult bloodstream infections.

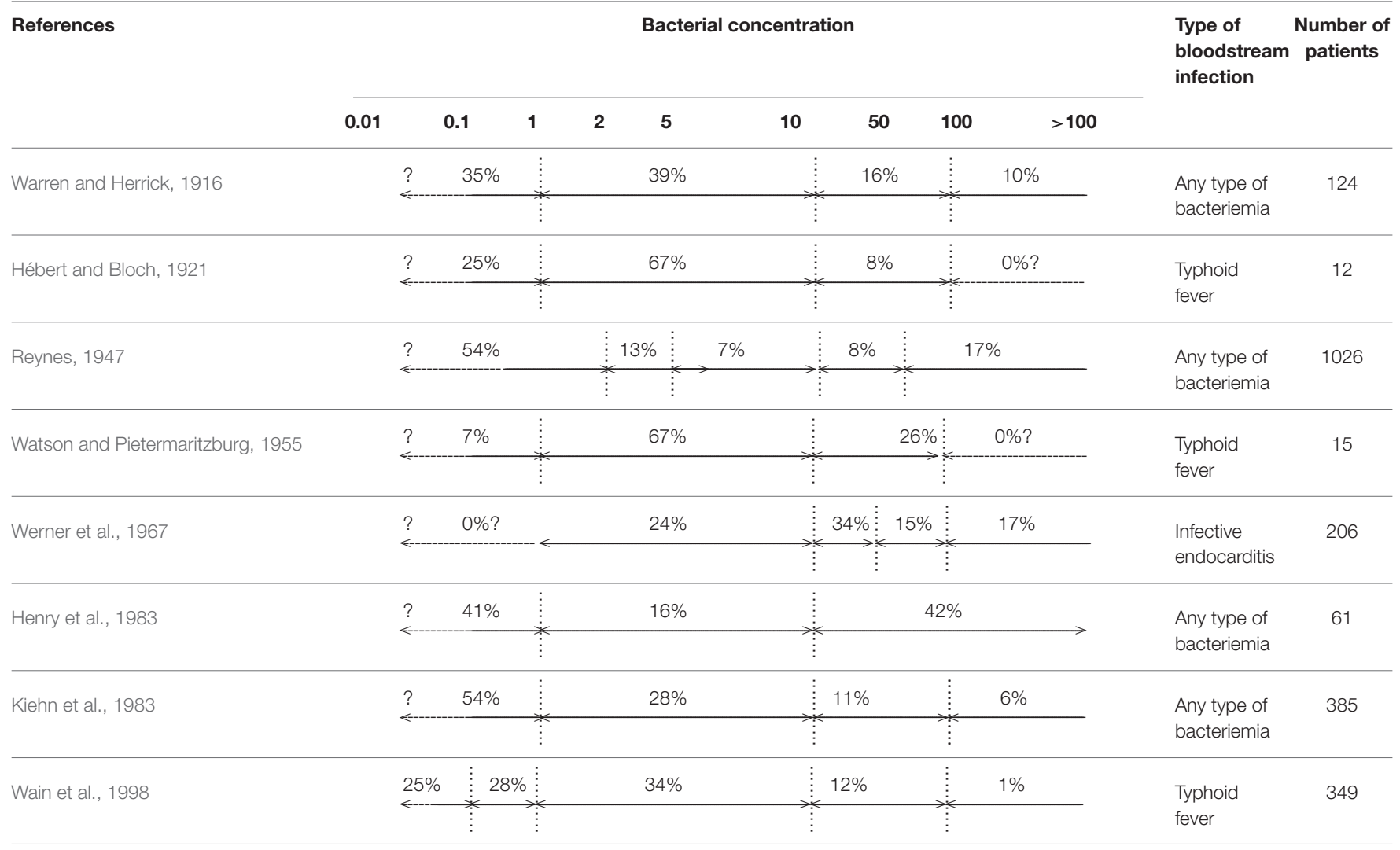

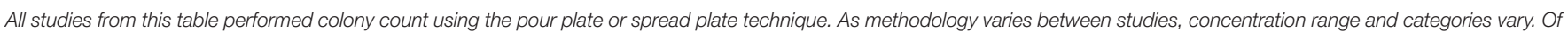
note, the quality and fertility of culture media may have varied between 1916 and 1998.

TABLE 2 | Fungal concentration in blood from bloodstream infection.

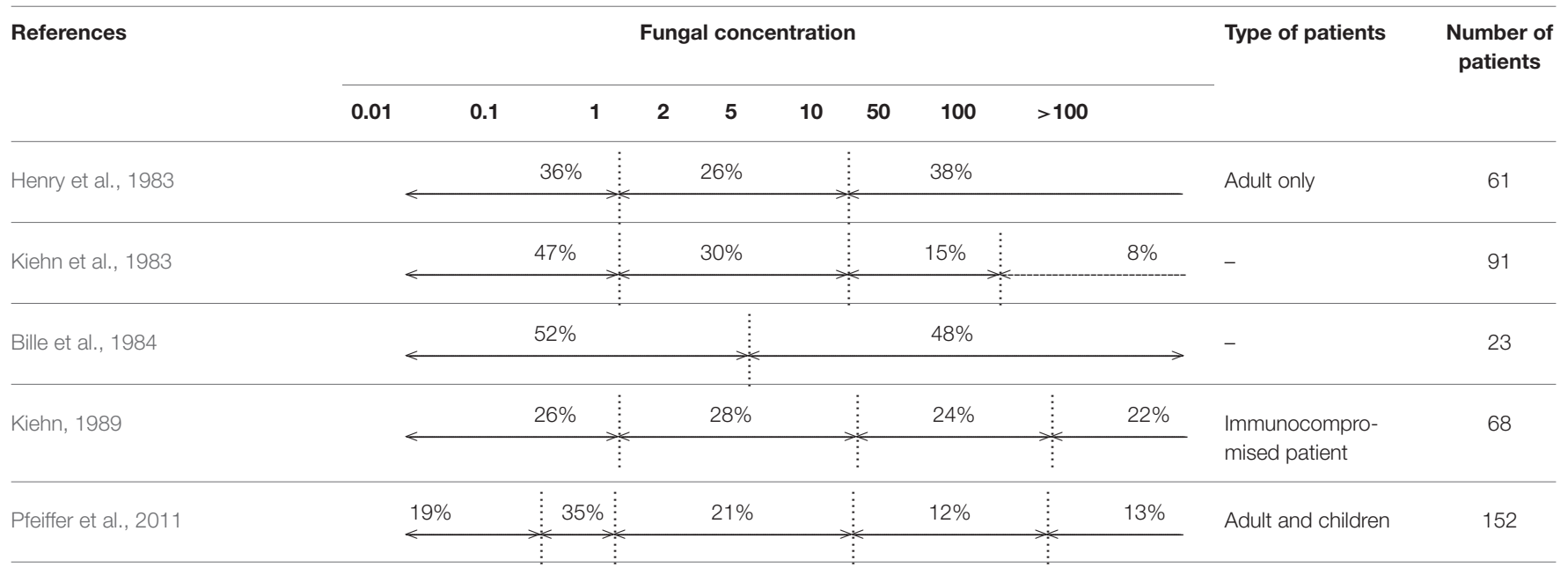

have long been similar (Baron et al., 2005b). Recent ASM/IDSA joint guidelines for adults recommend $20-30 \mathrm{~mL}$ of blood per culture set, which, according to these guidelines, may require more than 2 bottles depending on the system. Generally, in adults with a suspicion of BSI, 2-4 BC sets should be obtained in the evaluation of each septic episode (Baron et al., 2013; Dellinger et al., 2013).
For optimal recovery, each BC set should include paired aerobic and anaerobic bottles, the aerobic bottle being filled first. Besides, the culture of only 2 bottles (1 set) during a 24-h period from adult patients (hereafter referred to as "solitary BC"), is discouraged in all guidelines, as the sensitivity of only 2 bottles is insufficient. In addition, it must be taken into account that, in the real life, a significant proportion of BCs bottles are not 
adequately filled (Vitrat-Hincky et al., 2011; Willems et al., 2012; Lin et al., 2013; van Ingen et al., 2013; Coorevits and Van den Abeele, 2015). The "solitary BC" practice, still common, is not acceptable, due to the detrimental outcome when BSI is not detected and appropriately managed, in terms of antimicrobial treatment (a positive BC will impact on the selection of active anti-infective agents for the appropriate treatment duration), but also to trigger the identification, and eradication of the BSI source (Lamy and Seifert, 2012; Baron et al., 2013; Accoceberry et al., 2015a).

\section{Compliance with Guidelines and Current Caveats}

An increasing amount of data highlights the pre-analytical deficiencies of BC sampling which may compromise patient management by reducing BCs diagnostic yield (Schifman et al., 1991; Washington, 1992; Novis et al., 2001).

Firstly, BC bottles that should contain $8-10 \mathrm{~mL}$ of blood (manufacturer instructions) are frequently under-filled, consequently leading to sets of much less than $20 \mathrm{~mL}$, oralthough less commonly-over-filled $(>10 \mathrm{~mL})$. Mermel and Maki (1993) first highlighted the issue of under-filled BC bottles, a situation that was not center-specific, since $88 \%$ of 71 U.S. laboratories acknowledged that they routinely receive BC specimens from adults containing less than $5 \mathrm{~mL}$ of blood (Mermel and Maki, 1993). In Spain, in a prospective analysis conducted from 601 patients with suspected BSI, the mean volume of blood per BC bottle was estimated at $5 \mathrm{~mL}$ (Bouza et al., 2007). Similar sobering results have been reported from all over the world, with wide variations between countries (Table 3).

On the other hand, $\mathrm{BC}$ bottles are inoculated with more than $10 \mathrm{~mL}$ of blood in $7.6-13 \%$ of cases (Table 3). These bottles are at increased risk to be falsely flagged positive by the BC system (Wilson et al., 1994; Reimer et al., 1997). According to Willems et al. (2012), both Becton Dickinson ${ }^{\circledR}$ (BD) and bioMérieux ${ }^{\circledR}$ admitted that the vacuum in the BC bottles substantially exceeds the optimal blood fill volume (10 mL; Willems et al., 2012). The purpose of the vacuum excess is to ensure a sufficiently long expiration date, and to minimize the collection time. All these data underline how common are situations of non-compliance with manufacturer instructions, regarding the volume of blood to be inoculated for optimal diagnostic yield.

Secondly, BCs are commonly "solitary BC", for various reasons (e.g., resolution of fever, venipuncture failures, patients discharged home, or to another department where the ordering of BCs is discontinued, omission in collecting subsequent BC sets). Several studies reported rates of solitary BC per center between 10 and $33.3 \%$ (Table 4). Proportions of solitary BC seem to be lower in the US, with medians of $26 \%$, and $12.7 \%$ (Schifman et al., 1991; Novis et al., 2001), which may be related to the implementation of quality control programs that include this indicator, and to the fact that the personnel primarily responsible for $\mathrm{BC}$ collection are dedicated phlebotomists, two major differences with practices in most European countries. The combined effect of under-filled BC bottles and high proportion of solitary BCs, results in a high proportion of patients suspected of BSI for whom the total volume of blood inoculated is insufficient.
Given the critical importance of sampling the adequate amount of blood for BC sensitivity, monitoring the volume of blood cultured is a strong quality-assurance requirement (Mermel and Maki, 1993; Schifman et al., 1996; Willems et al., 2012; Chang et al., 2015; Accoceberry et al., 2015a). It should be surveyed through systematic program, either internal or external to the microbiology lab, and should at least include the proportions of solitary BC, and of adequately filled BC bottles (Schifman et al., 1991, 1996; Mermel and Maki, 1993; Novis et al., 2001; Willems et al., 2012; van Ingen et al., 2013; Chang et al., 2015; Accoceberry et al., 2015a). This latter may be estimated through either measurement of the weight of BC bottles received at the laboratories or visual inspection, although the latter is less accurate.

\section{Sampling Strategy}

Sampling an adequate volume of blood to ensure optimal sensitivity for BSI detection can be achieved either by increasing the number of venipunctures (hereafter, "multi-sampling strategy") or by collecting the adequate large volume through one single puncture (hereafter, "single-sampling strategy").

\section{Multi-Sampling Strategy \\ Rationale}

The multi-sampling strategy has been developed, and recommended for more than 40 years, and its practice has been generalized (Washington, 1975, 1992). The rationale of this strategy is based on the following points: (i) repetition of samples increases the total volume of blood cultured, thereby improving BC sensitivity, (ii) separate samples may discriminate contaminants from pathogens when BCs grow, (iii) separate samples improve BSI detection in case of intermittent bacteremia (Washington, 1975; Reimer et al., 1997).

It has been successful to improve BSI detection in several studies which concluded that, under routine circumstances, at least two separate sets of BCs should be sampled during a 24$\mathrm{h}$ period for the diagnosis of BSIs (Mermel and Maki, 1993; Li et al., 1994; Weinstein, 1996; Cockerill et al., 2004; Bouza et al., 2007; Lee et al., 2007), and guidelines recommend two to three or four - BC sets (O'Grady et al., 2008; Lamy and Seifert, 2012; Baron et al., 2013; Dellinger et al., 2013; Public Health England, 2014; Accoceberry et al., 2015a).

\section{Interpretation of positive culture results}

The differentiation between clinically significant positive BCs (i.e., the micro-organism identified is involved in the symptoms presented by the patient), and contaminants, is based on the number of positive BC samples when the culture yielded an organism regarded as a common contaminant [e.g., coagulase-negative staphylococci (CoNS), Corynebacterium spp, Micrococcus spp, etc.] (MacGregor and Beaty, 1972). For these microorganisms, at least 2 positive BCs yielding the same CoNS are usually required to consider the results as clinically significant, given that the likelihood that it represents true bacteremia as opposed to contamination increases with the number of positive bottles (Weinstein, 1996; Reimer et al., 1997; Kirn and Weinstein, 2013; Garcia et al., 2015). This can assist 


\begin{tabular}{|c|c|c|c|c|c|}
\hline \multirow[b]{2}{*}{ References } & \multicolumn{2}{|c|}{ Under-filled bottles } & \multicolumn{2}{|c|}{ Over-filled bottles } & \multirow[b]{2}{*}{ Country } \\
\hline & Threshold (mL) & Rate (\%) & Threshold (mL) & Rate (\%) & \\
\hline Vitrat-Hincky et al., 2011 & $<8$ & 65 & $>10$ & 13.0 & France \\
\hline Willems et al., 2012 a,b & $<8$ & $26.2-36.0$ & $>12$ & $7.6-12.8$ & Belgium \\
\hline van Ingen et al., 2013 & $<8$ & 55.3 & - & - & The Nederlands \\
\hline Coorevits and Van den Abeele, 2015 & $<8$ & 28.0 & $>12$ & 23.2 & Belgium \\
\hline Chang et al., 2015 & $<8$ & 97.7 & $>10$ & 0.2 & South Korea \\
\hline Lin et al., 2013 & $<7$ & 28.3 & $>10$ & 13.3 & Taiwan \\
\hline Mermel and Maki, 1993 & $<5$ & 20 & - & - & USA \\
\hline Chang et al., 2015 & $<3$ & 48.4 & - & - & South Korea \\
\hline
\end{tabular}

${ }^{a}$ Data from 5 hospitals

${ }^{b}$ Thresholds were defined as $2 \mathrm{~mL}$ below and above the recommended volume per vial.

TABLE 4 | Rate of solitary blood cultures.

\begin{tabular}{lcc}
\hline References & No. of institutions & Rate (\%) \\
\hline Gross et al., 1988 & 1 & 28.0 \\
Makadon et al., 1987 & 1 & 20.0 \\
Schifman et al., 1991 & 38 & 26.0 (median) \\
Schifman et al., 1996 & 909 & $10.1-12.1$ (inpatients) \\
& & $25.4-33.3$ (outpatients) \\
Novis et al., 2001 & 333 & 12.7 (median) \\
Vitrat-Hincky et al., 2011 & 1 & 28.0 \\
Neves et al., 2015 & 1 & 23.2 \\
\hline
\end{tabular}

in interpreting positive $\mathrm{BC}$ results, although the clinical context, including the presence of intravascular foreign devices, is also of paramount importance for an accurate interpretation of BCs positive for CoNS.

\section{Limitations}

Despite these recommendations, several issues have been highlighted: firstly, the proportion of solitary BCs is high with the multi-sampling strategy (Schifman et al., 1991; Vitrat-Hincky et al., 2011; Neves et al., 2015), and these are associated with a major default of sensitivity. In addition, solitary BC makes it more difficult to distinguish contaminants from pathogens. Secondly, each venipuncture required for the multisampling strategy is an additional opportunity for contamination (Aronson and Bor, 1987; Lamy et al., 2002; Patel et al., 2011). The contamination rate per draw has been estimated at $0.5-6 \%$ (Bates et al., 1991; Salluzzo and Reilly, 1991; Washington, 1992; Arendrup et al., 1996; Garcia et al., 2015). However, considering that only one positive sample may be interpreted as a confirmation that the patient has, indeed, BSI, and that the prevalence of BSI among patients suspected of BSI is relatively low, the proportion of false-positive among patients with positive BC may be as high as 20-56\% (Bates et al., 1991; Salluzzo and Reilly, 1991; Little et al., 1997). The consequences of BC contamination and false positives, although poorly studied, are not trivial, as they may lead to longer hospital stays, useless prescription of antibacterial or antifungal agents, additional investigations (e.g., echocardiography, repeated sampling for BC; Bates et al., 1991; Souvenir et al., 1998; Hall and Lyman, 2006; Gander et al., 2009; Alahmadi et al., 2011). In a retrospective case-control study on 254 false-positive BC results, Alahmadi et al. (2011) demonstrated that hospital length of stay increased by 5.4 days (2.8-8.1), with an additional hospital cost of $£ 1,270,381$ per year. In another study, contaminated BCs increased patient charges by $47 \%$ with an estimated cost of $\$ 8,720$ per contamination (Gander et al., 2009). Souvenir et al. (1998) reported that almost half of patients with positive BCs that were finally classified as "contaminants" were treated with antibiotics, including $34 \%$ by vancomycin. Additional costs were estimated at $\$ 1000$ per patient in this study, with a median increase in length of stay of 4.5 days (Souvenir et al., 1998). False-positive BCs generated a $20 \%$ increase in laboratory tests and a $39 \%$ increase in intravenous antibiotic charges in another study (Bates et al., 1991).

Finally, the theoretical concept of intermittent bacteremia or fungemia that supports the multi-sampling strategy has never been proved. Evidence suggests that most cases of clinically significant BSI are associated with continuous bacteremia or fungemia over a $24 \mathrm{~h}$ period, but with very low concentrations of circulating microorganisms (Jonsson et al., 1993; Li et al., 1994; Riedel et al., 2008).

\section{Single-Sampling Strategy Rationale}

The single-sampling strategy collects the total volume of blood from one single draw, a "BC set" of 4 to 6 bottles. This strategy satisfies both the need to collect a sufficient volume of blood, and the need to decrease contamination rate by limiting the number of punctures. In addition, this would be associated with decreased workload and risk of occupational exposure to blood-transmissible pathogens for nurses, decreased cost, and improved comfort for patients, by reducing the number of invasive, potentially painful, procedures. This strategy was developed since the late 1990s, based on the following: (i) the concept of intermittent bacteremia or fungemia may be erroneous (Jonsson et al., 1993; Li et al., 1994; Riedel et al., 2008); 
(ii) the key determinant for the capacity of BCs to diagnose BSI is the total volume of blood inoculated (Li et al., 1994); (iii) the rate of false-positive results increases with the number of draws; (iv) for a given volume of blood inoculated, the multi- and singlesampling strategies are expected to have similar sensitivity; (v) because the total volume is obtained at once with the singlesampling strategy, there is no risk of omitting subsequent draws, thereby eradicating the risk of solitary BC. Hence, the median total volume of blood inoculated will necessarily be greater; (vi) the single-sampling strategy should enable early initiation of empirical antibiotic treatment when indicated (e.g., severe sepsis), as there will be no need to postpone until subsequent sampling; and (vii) patient comfort will be improved, as only one venipuncture will be requested for this strategy.

First, as mentioned above, Li et al. (1994) demonstrated that increasing the volume of blood inoculated increases the yield of $\mathrm{BC}$, whether or not BCs are drawn simultaneously or serially within $24 \mathrm{~h}$ ( $\mathrm{Li}$ et al., 1994). To reconcile the interpretation of previous data (Washington, 1975; Cockerill et al., 2004) with these results, it has been hypothesized that the need to repeat BC sampling originated from the poor sensitivity when small volume of blood are inoculated ( $<20 \mathrm{~mL}$ per set). In such a procedure, the detection depends on the bacterial density at time of samplings, and not all samples will turn positive. Hence, when large volume of blood is inoculated on BC media (i.e., 40 or $60 \mathrm{~mL}$ ), the sensitivity of the single-sampling strategy is high whenever the sample is obtained and is similar to the sensitivity of multi-sampling strategy (Figure 1; Li et al., 1994; Lamy et al., 2002).

Arendrup et al. (1996) first evaluated the single-sampling strategy in their institution (Arendrup et al., 1996). Inoculating a single-sampling of $40-\mathrm{mL} \mathrm{BC} \mathrm{(4} \mathrm{bottles),} \mathrm{instead} \mathrm{of} 30-\mathrm{mL} \mathrm{BC}$ (3 bottles) increased the diagnostic yield of BC by $4.2 \%$. Using a before/after design, the authors concluded that this strategy had a positive impact on workload, and early initiation of empirical antimicrobial therapy. In most cases of positive BCs due to contaminants, only one or two of the four bottles turned positive, and the interpretation of positive BCs with a microorganism of questionable significance was not more difficult than with the multi-sampling strategy (Arendrup et al., 1996).

Two studies evaluated the impact of the number of draws on the risk of false-positive BCs (specificity), and quantified it using theoretical probabilistic approach (Aronson and Bor, 1987; Lamy et al., 2002). Both confirmed the negative impact of the number of separate sampling on BC specificity. One model-based study compared the performance (sensitivity and specificity) of the multi-sampling vs. the single-sampling strategies, using literature-based simulations and a quantitative risk-analysis approach. The median specificity of positive BCs decreased from 0.98 with the single-sampling strategy, to 0.91 with the 3 samplestrategy, which resulted, with a pretest probability of BSI set at $15 \%$, in a median positive predictive value decrease from 0.85 to 0.66 , respectively (Lamy et al., 2002). On the opposite, a 6 bottlecollection of blood at once (totalizing 35-42 mL) ensured an efficient diagnosis of bacterial BSI with an optimized specificity (Lamy et al., 2002). In this scenario, with a median volume of $7 \mathrm{~mL}$ of blood per bottle, the sensitivity was $\geq 0.95$ in $89 \%$

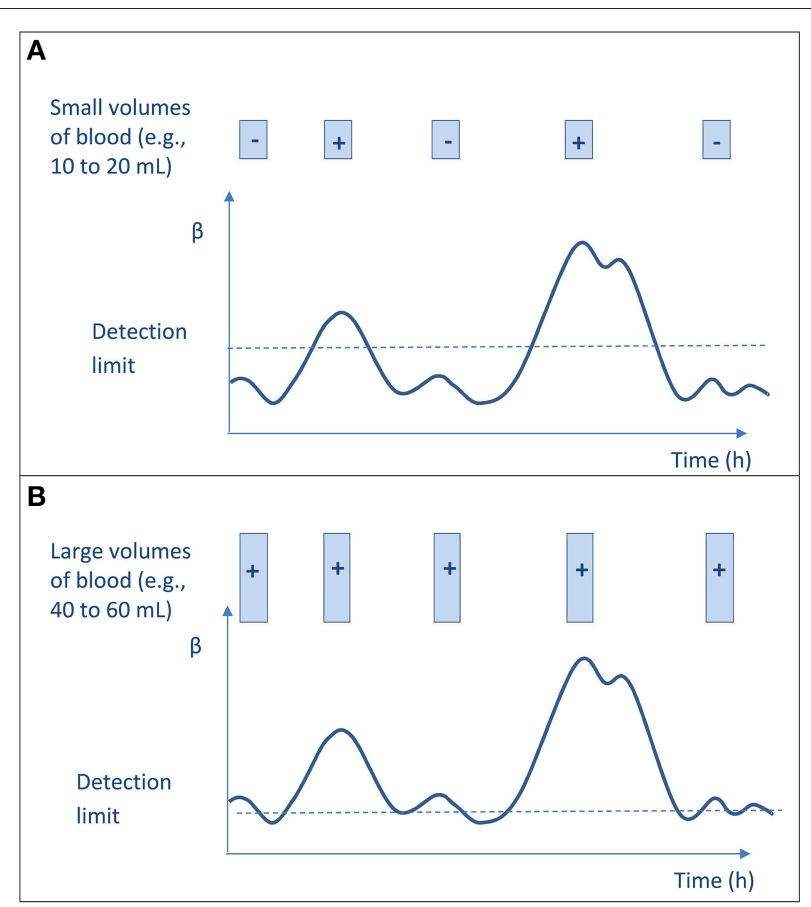

FIGURE 1 | Blood culture result (negative or positive) according to the amount of blood cultured at each sampling and to the microbial burden in blood. The curve represents the bacterial concentration ( $\beta$ ) in blood that varies with time and may be very low, but never null. The limit of bacteremia detection (BC sensitivity) is indicated with dotted line. Each sample is represented by a box. (A) Culturing low volumes of blood does not ensure sensitive testing and low detection threshold, and thus leads to uncertain bacteremia detection according to time of collection. The overall results suggest an intermittent bacteremia. (B) Culturing large volumes of blood ensures low detection threshold, thus allowing detecting bacteremia whenever the sample is obtained. One sample is enough for confidently detecting bacteremia; the overall results would suggest a continuous bacteremia.

of the trials, and the median specificity was $97.5 \%$. Recently, a prospective study performed in the adult emergency departments of three university hospitals compared a single-sampling strategy collecting one large volume of blood (4 bottles), to the standard multi-sampling strategy. Overall, the single-sampling strategy allowed detection of pathogens in blood of $97.4 \%$ of patients vs. $95.5 \%$ for the multi-sampling strategy. In the subgroup of patients for whom two sets were drawn, the single-sampling strategy was superior to the multi-sampling strategy in term of positive results. In addition, considering the overall performances (sensitivity and specificity), the single-sampling strategy was significantly better than the multi-sampling strategy (Dargère et al., 2014). Finally, as expected, the proportion of solitary BC rate was strikingly reduced by using the single-sampling strategy.

Although this strategy has been approved in France as an alternative to the multi-sampling strategy since 2007 (SFM, 2007) a nationwide survey conducted in 47 hospitals found that only 17 and $21 \%$ of them were using the single-sampling strategy in 2013, exclusively and partly, respectively (Royer et al., 2015). In Denmark, where the single-sampling strategy first spread, a survey on BC practices was conducted in 2015 among 6/11 
departments of clinical microbiology, covering half of Danish hospital admissions (1.28/2.57 millions admission). Responses showed that 1 out of 6 departments practiced the multi-sampling strategy, 2 practiced either multi-sampling or single-sampling strategy, and 3 hospitals practiced the single-sampling strategy. Five of these centers use the multi-sampling strategy when endocarditis is suspected.

\section{Interpretation of positive $B C$ results}

Rules for interpretation of positive BC results, and distinction between BSI and contamination, differ with the single-sampling strategy, as the information given by the proportion of positive $\mathrm{BC}$ sets at different times cannot be used. In order to define guideline to differentiate clinically significant bacteria from contaminants with this strategy, a study was conducted between 2007 and 2008 in Lyon University Hospital (France), where the single-sampling strategy has been implemented in 2004 (Leyssene et al., 2011). In monomicrobial positive sets (one set being defined as 6 bottles originating from a single venipuncture), the positive predictive value (PPV) of BC was 88 and 100\% with, respectively, one and $\geq 2$ positive bottles for $E$. coli, while it was $100 \%$ for S. aureus, Pseudomonas aeruginosa, and Candida spp., whatever the number of positive bottles. For CoNS, the PPV with one, two, three or $\geq 4$ positive bottle(s) was 3.5, 61.1, 78.9, and $100 \%$, respectively. The most difficult cases to interpret were those with 2 or 3 positive bottles out of the 6 cultured, but this was the case for only $5 \%$ of patients with CoNS-growing BC. Therefore, despite systematic evaluations of an approach based on the number of positive bottles have proven to be unreliable when using the multi-sampling strategy (Mirrett et al., 2001b; Kirn and Weinstein, 2013), the clinical significance of CoNS-growing BC was correlated with the number of positive bottles when using the single-sampling strategy (Leyssene et al., 2011). Similar findings were observed in Arendrup et al. study (Arendrup et al., 1996). Consistent with these data, Dargère et al. (2014) showed that most contaminants were detected only in the first aerobic bottle of the 4-bottle set with the single-sampling strategy, and that the most frequent microorganism was CoNS (Dargère et al., 2014).

\section{Limitations}

Firstly, getting a sufficient volume of blood to fill 6 bottles from a single venipuncture may be difficult, particularly in the elderly, and in patients with shock. This may require an additional puncture to collect the total volume of blood necessary for optimal diagnostic yield of BCs. Secondly, the level of evidence on the performance of the single-sampling strategy remains low: only two published studies evaluated the performances of a single-sampling strategy based on inoculating $40 \mathrm{~mL}$ of blood (Arendrup et al., 1996; Dargère et al., 2014). The first one was a quasi-experimental study (i.e., before/after design), and the second one was randomized, but limited by sample size $(n=$ 2374). Indeed, this lack of studies is mainly due to the fact that comparative studies are difficult to perform as they require a complex design to prevent biases, large sample sizes to be adequately powered, and a strict methodology to control the volume of blood actually cultured in each group. Thirdly, level of evidence is also lacking for specific situations such as infective endocarditis (IE) - for which the number of positive samples on distinct venipunctures is part of the modified Duke Criteria ( $\mathrm{Li}$ et al., 2000)—or for CLABSI. A high BC sensitivity is expected for detecting IE with the single-sampling strategy because of the high density of bacteria in blood (Werner et al., 1967), but modified Duke Criteria would require to be adapted if the singlesampling strategy was to be generalized. In patients suspected of CLABSI, the diagnosis may be obtained through a singlesampling strategy (4-6 bottles) obtained through venipuncture, associated with one appropriately filled bottle simultaneously drawn from the catheter line to be able to estimate the differential time to positive BC (DTTP). Indeed, despite one peripheral $\mathrm{BC}$ set has been shown to be appropriate for the DTTP-based diagnosis of CLABSI (Mermel et al., 2009; Guembe et al., 2012), a 4-6 bottle set drawn by venipuncture is still indicated for the diagnosis of BSI, whatever the source, including thus BSI other than CLABSI. However, such protocols need to be investigated.

In addition, to the best of our knowledge, the medicoeconomic aspects of these two strategies have not been thoroughly evaluated. Other potential impacts of the singlesampling strategy remain to be measured, including patient comfort (e.g., only one venipuncture with the singlesampling strategy), the risk of occupational-exposure to blood-transmissible pathogens, and the timing of empirical anti-infective agent initiation.

\section{BLOOD CULTURE SYSTEMS}

In this review, considerations on BC systems will be limited to those that may impact the performance (sensitivity, specificity) of BSI diagnosis. Overall, three continuous-monitoring automated BC systems are available in 2015. The two system most commonly used are, by far, the Bactec (Beckton-Dickinson, USA) and BactAlert/Virtuo systems (bioMérieux, France). These two systems are based on the utilization of carbohydrate substrates in culture media, and subsequent production of $\mathrm{CO}_{2}$ by growing microorganisms, detected through their impact on $\mathrm{pH}$, by either a colorimetric, or a fluorescent sensor placed at the bottom of the bottle. Both systems have similar characteristics, and, despite differences in culture media, they share similar performances for detection of microorganisms (Kirn and Weinstein, 2013). The fertility of the culture media used in these systems is among the highest among broth media available in clinical microbiology. Media are combined in sets in order to include aerobic and anaerobic formulations, and those associated with resins or charcoal regularly show better performances as compared to standard media (Weinstein et al., 1995; Wilson et al., 1995, 2001; Gibb et al., 1998; Mirrett et al., 2004). Only slight improvements in the performances have been achieved over the last 15 years (e.g., Kirn et al., 2014).

Combined with inadequate sampling volumes, the early use of antibiotics prior to the first blood sample is a critical factor hampering microorganism recovery. Some media include resin or charcoal to neutralize antibiotics. The superior neutralizing capacity of resin-based media over the bioMérieux charcoal 
medium has been demonstrated (Flayhart et al., 2007), but discrepancies have been reported and the complete neutralization might be insufficient. The types and compositions of resins, the type of antibiotics and the MICs of the test strains may account for the differences in neutralization performance (Mitteregger et al., 2013). As far as possible, BCs should be obtained before the antimicrobial therapy is initiated.

\section{False-Positive Instrument Signal}

False-positive instrument signal, defined as a bottle flagged positive by the system though not containing any microorganism, has been described with the systems, in less than $1 \%$ of bottles (Pohlman et al., 1995; Mirrett et al., 2001a). These falsepositive bottles require quick handle and re-incubation into the $\mathrm{BC}$ system (e.g., within $1 \mathrm{~h}$ following their unloading) in order to resume the bottle analysis. Major causes of false-positive results include high level of leukocyte counts, over-filled bottles and/or errors in incubation so that the bottles are monitored with an inappropriate algorithm (Wilson et al., 1994; Reimer et al., 1997). However, available data are scarce and mostly derive from studies of media that are not in use anymore. Given the significant changes in media formulation over years, updated evaluations are required to evaluate the actual incidence of false-positive signals, and the effect of over-filled bottles on positivity.

\section{False-Negative Instrument Signal}

False-negative instrument signal is defined as a bottle flagged negative by the system although it contains bacteria or fungi. The main parameter associated with false-negative signal is the preincubation delay, which is the time difference between the time point of bottle loading in the $\mathrm{BC}$ incubator and the time point of bottles filling. It has been estimated that 0.3 to $15.3 \%$ of bottles containing bacteria or fungi are flagged negative by BC system (Reimer et al., 1997; Klaerner et al., 2000; Mirrett et al., 2001a; Lemming et al., 2004; Seegmüller et al., 2004), although few of the media currently in use have been evaluated for this default. The false-negative signal depends on the temperature at which the bottles were kept before loading (hereafter pre-incubation temperature), the pre-incubation duration, the type of microorganism involved, and the pairing instrument/type of bottle used. Overall, the false negative signal rate was greater when pre-incubation temperature was $35^{\circ} \mathrm{C}$, pre-incubation duration was $>24 \mathrm{~h}$, with streptococci, Candida sp, or Pseudomonas sp, and with Bactec systems (Sautter et al., 2006). A pre-incubation duration $<12 \mathrm{~h}$ kept the risk of false negative at its minimum (Lemming et al., 2004; Seegmüller et al., 2004; Akan and Yildiz, 2006; Sautter et al., 2006). It has been hypothesized that microorganisms grow in the bottle during the pre-incubation duration, especially with high temperature $\left(35^{\circ} \mathrm{C}\right)$, so that they have already reached the stationary phase at time of loading. In such a situation, positive bottles cannot be detected by the instrument algorithms. Only $60 \%$ of false-negative signals were detected by visual inspection before loading (Lemming et al., 2004). Preincubation at $35^{\circ} \mathrm{C}$ should be discouraged (Lamy and Seifert, 2012; Baron et al., 2013; Kirn and Weinstein, 2013; Accoceberry et al., 2015a), and suppliers have revised their guidelines in this way. Indeed, bottles should be delivered to the laboratory and loaded in the BC system as fast as possible (Seegmüller et al., 2004). This point is pivotal especially as studies on BC transport time have shown that delayed entry negatively impacts on time to positivity from the time point of sampling (Ronnberg et al., 2013) or on the positive BC rate (Morton et al., 2015). Indeed, Morton et al. (2015) highlighted that BC yield was lower when BCs were collected during the week-end, and this was likely caused by delays or errors in incubation and processing, in relation with the reduced provision of support services during the week-ends (Morton et al., 2015).

\section{Monitoring the Volume of Blood Inoculated}

As underlined, a major cause for missing a diagnosis of BSI is insufficient filling of $\mathrm{BC}$ bottles, which leads to a sub-optimal sensitivity. Improvement of BCs bottle filling would require enhanced training of phlebotomists, including systematic visual inspection of bottle filling at the bedside (van Ingen et al., 2013). In addition, some BC system manufacturers developed automated systems to estimate the level of bottle filling at the time of loading (Chang et al., 2015). The Bactec FX system (BD, USA), based on red blood cells metabolism, provides reliable estimates of the blood volume in $\mathrm{BC}$ bottles, with a mean error of $0.2 \mathrm{~mL}$. The error margin is higher (i.e., $1 \mathrm{~mL}$ ), for BC bottles received from hematology wards, due to anemia or impaired red blood cells metabolism (Chang et al., 2015). One limitation of this device is that volume is monitored in batches and not for each bottle individually. A device developed on the new BC system Virtuo (bioMerieux) provides an automated volume estimation of each bottle based on a photometric detection of liquid level, but, as far as we know, its accuracy has not yet been evaluated in clinical studies.

\section{Incubation Time}

Bottles are usually incubated for a maximum duration of 57 days (Lamy and Seifert, 2012; Baron et al., 2013; Kirn and Weinstein, 2013; Accoceberry et al., 2015a). Very few microorganisms are recovered between 5 and 7 days. Among these, clinically significant bacteria or fungi are mostly low-level $\mathrm{CO}_{2}$ producers, or slowly growing micro-organisms (e.g., Candida spp, strict anaerobes, or Brucella sp). Marginson et al. (2014) found that $2.7 \%$ of all positive $\mathrm{BC}$ turned positive between 5 and 7 days, including $0.5 \%$ of clinically significant microorganism (Marginson et al., 2014). When infective endocarditis is suspected but $\mathrm{BC}$ remain negative on day 5 , some guidelines state that bottles incubation must be prolonged until day 15 or more (Mainardi and Ricardo, 2012; Accoceberry et al., 2015b). However, these recommendations are mostly based on data originating from studies performed before the use of automated BC systems and highly-efficient culture media (Petti et al., 2006). Studies based on continuous-monitoring BC systems have shown that prolonged incubation time does not significantly improve the overall sensitivity of BC, even for IE due to fastidious bacteria from the HACEK group (Haemophilus, Aggregibacter, Cardiobacterium, Eikenella, Kingella) as all of these were recovered from a standard 5-day incubation protocol in various studies (Wilson et al., 1993; Baron et al., 2005a; Petti et al., 2006). Prolonged cultures of specific BC bottles for 
the detection of fastidious zoonotic agents in blood culturenegative endocarditis (BCNE) are exceptionally useful, and are not recommended in routine practice. Fournier et al. (2010) have demonstrated that serological analysis was the most useful specimen providing a diagnosis of $47.7 \%$ of 745 tested patients with suspected BCNE (mainly Q fever and Bartonella infections; Fournier et al., 2010). According to the local epidemiology, the diagnosis of BCNE is based on systematic serological testing for Coxiella burnetii, Bartonella spp., Brucella spp., Aspergillus spp., Mycoplasma pneumonia, Legionella pneumophila followed by specific polymerase chain reaction (PCR) assays (Tropheryma whipplei, Bartonella spp. and fungi) (Baddour et al., 2015; Habib et al., 2015).

\section{CONCLUSIONS}

BCs are among the most common microbiological tests performed in 2016 and remain the first and essential diagnostic tool for detection of BSIs. Best practices of BC sampling require thorough understanding of several issues including appropriate ordering $\mathrm{BCs}$, timing of $\mathrm{BC}$ collection, skin preparation, sample site, impact of the volume sampled. Quality control programs,

\section{REFERENCES}

Accoceberry, I., Cornet, M., Fenollar, F., and Mainardi, J. L. (2015b). "Endocardite," in REMIC, Référentiel en Microbiologie Médicale, $5^{\text {th }}$ Edn., eds T. Bourlet, R. Courcol, P. Laudat, J. L. Herrmann, L. Lachaud, B. Lamy, H. Peigue-Lafeuille, and B. Pangon (Paris: Société française de Microbiologie), 139-144.

Accoceberry, I., Cornet, M., and Lamy, B. (2015a). "Hémoculture," in REMIC, Référentiel en Microbiologie Médicale, $5^{\text {th }}$ Edn., eds T. Bourlet, R. Courcol, P. Laudat, J. L. Herrmann, L. Lachaud, B. Lamy, H. Peigue-Lafeuille, and B. Pangon (Paris: Société française de Microbiologie), 125-137.

Akan, O. A., and Yildiz, E. (2006). Comparison of the effect of delayed entry into 2 different blood culture systems (BACTEC 9240 and BacT/ALERT 3D) on culture positivity. Diagn. Microbiol. Infect. Dis. 54, 193-196. doi: 10.1016/j.diagmicrobio.2005.09.016

Alahmadi, Y. M., Aldeyab, M. A., McElnay, J. C., McElnay, J. C., Scott, M. G., Darwish, F. W., et al. (2011). Clinical and economic impact of contaminated blood cultures within the hospital setting. J. Hosp. Infect. 77, 233-236. doi: 10.1016/j.jhin.2010.09.033

Arendrup, M., Jensen, I. P., and Justesen, T. (1996). Diagnosing bacteremia at a Danish hospital using one early large blood volume for culture. Scand. J. Infect. Dis. 28, 609-614.

Aronson, M. D., and Bor, D. H. (1987). Blood cultures. Ann. Intern. Med. 106, 246-253.

Arpi, M., Bentzon, M. W., Jensen, J., and Frederiksen, W. (1989). Importance of blood volume cultured in the detection of bacteremia. Eur. J. Clin. Microbiol. Infect. 8, 838-842.

Baddour, L. M., Wilson, W. R., Bayer, A. S., Fowler, V. G. Jr., Tleyjeh, I. M., Rybak, M. J., et al. (2015). Infective endocarditis in adults: diagnosis, antimicrobial therapy, and management of complications: a scientific statement for healthcare professionals from the american heart association. Circulation 132, 1435-1486. doi: 10.1161/CIR.0000000000000296

Baron, E. J., Miller, J. M., Weinstein, M. P., Richter, S. S., Gilligan, P. H., Thomson, R. B. Jr., et al. (2013). A guide to utilization of the microbiology laboratory for diagnosis of infectious diseases: 2013 recommendations by the Infectious Diseases Society of America (IDSA) and the American Society for Microbiology (ASM) (a). Clin. Infect. Dis. 57, e22-e121. doi: 10.1093/cid/cit278

Baron, E. J., Scott, J. D., and Tompkins, L. S. (2005a). Prolonged incubation and extensive subculturing do not increase recovery of clinically significant including automated controls of pre-analytical variables should be reinforced to address the deficiencies that are frequently reported.

While both sampling strategies (i.e., multi-sampling strategy, or single-sampling strategy) are acceptable according to the guidelines, additional progresses are expected with the singlesampling strategy that is probably more convenient for patients and healthcare workers and probably associated with reduced costs, earlier initiation of empirical anti-infective treatment when indicated, and lower risk of occupational exposure to bloodtransmissible pathogens. Today, evidence-based clinical data to document the yield of this strategy deserve to be improved and a large multicenter randomized trial is required to decipher the pro and cons of both strategies of $\mathrm{BC}$ collection for the diagnosis of BSIs and infective endocarditis.

\section{AUTHOR CONTRIBUTIONS}

Conceived and designed the work: JJP, SD, BL, and PT. Performed survey: MC. Drafted the paper: BL, SD, and PT. Critically revised the manuscript: PT, MA. All authors read and approved the final manuscript. microorganisms from standard automated blood cultures. Clin. Infect. Dis. 41, 1677-1680. doi: 10.1086/497595

Baron, E. J., Weinstein, M. P., Dunne, W. M., Yagupsky, P., Welch, D. F., and Wilson, D. M. (2005b). Cumitech 1C, Blood Cultures IV. Washington, DC: ASM Press.

Bates, D. W., Cook, E. F., Goldman, L., and Lee, T. H. (1990). Predicting bacteremia in hospitalized patients. a prospectively validated model. Ann. Intern. Med. 113, 495-500.

Bates, D. W., Goldman, L., and Lee, T. H. (1991). Contaminant blood cultures and resource utilization. the true consequences of false-positive results. JAMA 265, 365-369.

Bille, J., Edson, R. S., and Roberts, G. D. (1984). Clinical evaluation of the lysis-centrifugation blood culture system for the detection of fungemia and comparison with a conventional biphasic broth blood culture system. J. Clin. Microbiol. 19, 126-128.

Bouza, E., Sousa, D., Rodriguez-Creixems, M., Lechuz, J. G., and Munoz, P. (2007). Is the volume of blood cultured still a significant factor in the diagnosis of bloodstream infections? J. Clin. Microbiol. 45, 2765-2769. doi: 10.1128/JCM.00140-07

Boyce, J. M., Nadeau, J., Dumigan, D., Miller, D., Dubowsky, C., Reilly, L., et al. (2013). Obtaining blood cultures by venipuncture versus from central lines: impact on blood culture contamination rates and potential effect on central line-associated bloodstream infection reporting. Infect. Control. Hosp. Epidemiol. 34, 1042-1047. doi: 10.1086/673142

Caldeira, D., David, C., and Sampaio, C. (2011). Skin antiseptics in venous puncture-site disinfection for prevention of blood culture contamination: systematic review with meta-analysis. J. Hosp. Infect. 77, 223-232. doi: 10.1016/j.jhin.2010.10.015

Chang, J., Park, J. S., Park, S., Choi, B., Yoon, N. S., Sung, H., et al. (2015). Impact of monitoring blood volume in the BD BACTEC ${ }^{\mathrm{TM}}$ FX blood culture system: virtual volume versus actual volume. Diagn. Microbiol. Infect. Dis. 81, 89-93. doi: 10.1016/j.diagmicrobio.2014.11.001

Coburn, B., Morris, A. M., Tomlinson, G., and Detsky, A. S. (2012). Does this adult patient with suspected bacteremia require blood cultures? JAMA 308, 502-511. doi: $10.1001 /$ jama.2012.8262

Cockerill, F. R. III, Wilson, J. W., Vetter, E. A., Goodman, K. M., Torgerson, C. A., Harmsen, W. S., et al. (2004). Optimal testing parameters for blood cultures. Clin. Infect. Dis. 38, 1724-1730. doi: 10.1086/421087 
Coorevits, L., and Van den Abeele, A. M. (2015). Evaluation of the BD BACTEC FX blood volume monitoring system as a continuous quality improvement measure. Eur. J. Clin. Microbiol. Infect. Dis. 34, 1459-1466. doi: 10.1007/s10096015-2373-2

Dargère, S., Parienti, J. J., Roupie, E., Gancel, P. E., Wiel, E., Smaiti, N., et al. (2014). Unique blood culture for diagnosis of bloodstream infections in emergency departments: a prospective multicentre study. Clin. Microbiol. Infect. 20, O920O927. doi: 10.1111/1469-0691.12656

Dawson, S. (2014). Blood culture contaminants. J. Hosp. Infect. 87, 1-10. doi: 10.1016/j.jhin.2014.02.009

Dellinger, R. P., Levy, M. M., Rhodes, A., Annane, D., Gerlach, H., Opal, S. M., et al. (2013). Surviving sepsis campaign: international guidelines for management of severe sepsis and septic shock, 2012. Intensive Care Med. 39, 165-228. doi: $10.1007 /$ s00134-012-2769-8

Eliakim-Raz, N., Bates, D. W., and Leibovici, L. (2015). Predicting bacteraemia in validated models - a systematic review. Clin. Microbiol. Infect. 21, 295-301. doi: 10.1016/j.cmi.2015.01.023

Flayhart, D., Borek, A. P., Wakefield, T., Dick, J., and Carroll, K. C. (2007). Comparison of BACTEC PLUS blood culture media to BacT/Alert FA blood culture media for detection of bacterial pathogens in samples containing therapeutic levels of antibiotics. J. Clin. Microbiol. 45, 816-821. doi: 10.1128/JCM.02064-06

Fournier, P. E., Thuny, F., Richet, H., Lepidi, H., Casalta, J. P., Arzouni, J. P., et al. (2010). Comprehensive diagnostic strategy for blood culture-negative endocarditis: a prospective study of 819 new cases. Clin. Infect. Dis. 15, 131-140. doi: $10.1086 / 653675$

Gander, R. M., Byrd, L., DeCrescenzo, M., Hirany, S., Bowen, M., and Baughman, J. (2009). Impact of blood cultures drawn by phlebotomy on contamination rates and health care costs in a hospital emergency department. J. Clin. Microbiol. 47, 1021-1024. doi: 10.1128/JCM.02162-08

Garcia, R. A., Spitzer, E. D., Beaudry, J., Beck, C., Diblasi, R., Gilleeny-Blabac, M., et al. (2015). Multidisciplinary team review of best practices for collection and handling of blood cultures to determine effective interventions for increasing the yield of true-positive bacteremias, reducing contamination, and eliminating false-positive central line-associated bloodstream infections. Am. J. Infect. Control. 43, 1222-1237. doi: 10.1016/j.ajic.2015.06.030

Gibb, A. P., Hill, B., and Chorel, B. (1998). Comparative study of BacT/ALERT FAN bottles and Standard BacT/ALERT bottles. Diagn. Microbiol. Infect. Dis. 32, 159-163.

Goto, M., and Al Hasan, N. (2013). Overall burden of bloodstream infection and nosocomial bloodstream infection in North America and Europe. Clin. Microbiol. Infect. 19, 501-509. doi: 10.1111/1469-0691.12195

Gross, P. A., Van Antwerpen, C. L., Hess, W. A., and Reilly, K. A. (1988). Use and abuse of blood cultures: program to limit use. Am. J. Infect. Control 16, 114-117.

Guembe, M., Rodríguez-Créixems, M., Sánchez-Carrillo, C., Martín-Rabadán, P., and Bouza, E. (2012). Differential time to positivity (DTTP) for the diagnosis of catheter-related bloodstream infection: do we need to obtain one or more peripheral vein bloodcultures? Eur. J. Clin. Microbiol. Infect. Dis. 31, 1367-1372. doi: 10.1007/s10096-011-1451-3

Habib, G., Lancellotti, P., Antunes, M. J., Bongiorni, M. G., Casalta, J. P., Del Zotti, F., et al. (2015). 2015 ESC Guidelines for the management of infective endocarditis: the task force for the management of infective endocarditis of the European Society of Cardiology (ESC). Endorsed by: European Association for Cardio-Thoracic Surgery (EACTS), the European Association of Nuclear Medicine (EANM). Eur. Heart J. 36, 3075-3128. doi: 10.1093/eurheartj/ehv319

Hall, K. K., and Lyman, J. A. (2006). Updated review of blood culture contamination. Clin. Microbiol. Rev. 19, 788-802. doi: 10.1128/CMR.00062-05

Hébert, P., and Bloch, M. (1921). Recherches sur la fièvre typhoïde: Juillet 1915Janvier 1919. Ann. Inst. Pasteur 30, 157-208.

Henry, N. K., McLimans, C. A., Wright, A. J., Thompson, R. L., Wilson, W. R., and Washington, J. A. (1983). Microbiological and clinical evaluation of the isolator lysis-centrifugation blood culture tube. J. Clin. Microbiol. 17, 864-869.

Jonsson, B., Nyberg, A., and Henning, C. (1993). Theoretical aspects of detection of bacteraemia as a function of the volume of blood cultured. APMIS 101, 595-601.

Kaasch, A. J., Rieg, S., Hellmich, M., Kern, W. V., and Seifert, H. (2014). Differential time to positivity is not predictive for central line-related Staphylococcus aureus bloodstream infection in routine clinical care. J. Infect. 68, 58-61. doi: 10.1016/j.jinf.2013.08.006

Kiehn, T. E. (1989). Bacteremia and fungemia in the immunocompromised patient. Eur. J. Clin. Microbiol. Infect. Dis. 8, 832-837.

Kiehn, T. E., Wong, B., Edwards, F. F., and Armstrong, D. (1983). Comparative recovery of bacteria and yeasts from lysis-centrifugation and a conventional blood culture system. J. Clin. Microbiol. 18, 300-304.

Kirn, T. J., Mirrett, S., Reller, L. B., and Weinstein, M. P. (2014). Controlled clinical comparison of BacT/alert FA plus and FN plus blood culture media with BacT/alert FA and FN blood culture media. J. Clin. Microbiol. 52, 839-843. doi: 10.1128/JCM.03063-13

Kirn, T. J., and Weinstein, M. P. (2013). Update on blood cultures: how to obtain, process, report, and interpret. Clin. Microbiol. Infect. 19, 513-520. doi: 10.1111/1469-0691.12180

Klaerner, H. G., Eschenbach, U., Kamereck, K., Lehn, N., Wagner, H., and Miethke, T. (2000). Failure of an automated blood culture system to detect nonfermentative gram-negative bacteria. J. Clin. Microbiol. 38, 1036-1041.

Lamy, B., Roy, P., Carret, G., Flandrois, J. P., and Delignette-Muller, M. L. (2002). What is the relevance of obtaining multiple blood samples for culture? A comprehensive model to optimize the strategy for diagnosing bacteremia. Clin. Infect. Dis. 35, 842-850. doi: 10.1086/342383

Lamy, B., and Seifert, H. (2012). "Microbial diagnosis: septicemia," in European Manual of Clinical Microbiology (SFM/ESCMID), 1st Edn., eds G. Cornaglia, R. Courcol, J. L. Herrmann, G. Kahlmeter, H. Peigue-Lafeuille, and J. Vila (Paris: SFM), 101-110.

Lee, A., Mirrett, S., Reller, L. B., and Weinstein, M. P. (2007). Detection of bloodstream infections in adults: how many blood cultures are needed? J. Clin. Microbiol. 45, 3546-3548. doi: 10.1128/JCM.01555-07

Lemming, L., Holt, H. M., Petersen, I. S., Østergaard, C., and Bruun, B. (2004). Bactec 9240 blood culture system: to preincubate at 35 degrees $\mathrm{C}$ or not? Clin. Microbiol. Infect. 10, 1089-1091. doi: 10.1111/j.1469-0691.2004.00969.x

Levin, P. D., Moss, J., Stohl, S., Fried, E., Cohen, M. J., Sprung, C. L., et al. (2013). Use of the nonwire central line hub to reduce blood culture contamination. Chest 143, 640-645. doi: 10.1378/chest.12-0863

Leyssene, D., Gardes, S., Vilquin, P., Flandrois, J. P., Carret, G., and Lamy, B. (2011). Species-driven interpretation guidelines in case of a single-sampling strategy for blood culture. Eur. J. Clin. Microbiol. Infect. Dis. 30, 1537-1541. doi: 10.1007/s10096-011-1257-3

Li, J., Plorde, J. J., and Carlson, L. G. (1994). Effects of volume and periodicity on blood cultures. J. Clin. Microbiol. 32, 2829-2831.

Li, J. S., Sexton, D. J., Mick, N., Nettles, R., Fowler, V. G. Jr., Ryan, T., et al. (2000). Proposed modifications to the Duke criteria for the diagnosis of infective endocarditis. Clin. Infect. Dis. 30, 633-638. doi: 10.1086/313753

Lin, H. H., Liu, Y. F., Tien, N., Ho, C. M., Hsu, L. N., and Lu, J. J. (2013). Evaluation of the blood volume effect on the diagnosis of bacteremia in automated blood culture systems. J. Microbiol. Immunol. Infect. 46, 48-52. doi: 10.1016/j.jmii.2012.03.012

Little, J. R., Trovillion, E., and Fraser, V. (1997). High frequency of pseudobacteremia at a university hospital. Infect. Control. Hosp. Epidemiol. 18, 200-202.

MacGregor, R. R., and Beaty, H. N. (1972). Evaluation of positive blood cultures: guidelines for early differentiation of contaminated from valid positive cultures. Arch. Intern. Med. 130, 84-87.

Mainardi, J. L., and Ricardo, U. (2012). "Microbial diagnosis: endocarditis," in European Manual of Clinical Microbiology (SFM/ESCMID), 1st Edn., eds G. Cornaglia, R. Courcol, J. L. Herrmann, G. Kahlmeter, H. Peigue-Lafeuille, and J. Vila (Paris: SFM), 111-114

Makadon, H. J., Bor, D., Friedland, G., Dasse, P., Komaroff, A. L., and Aronson, M. D. (1987). Febrile inpatients: house officers' use of blood cultures. J. Gen. Intern. Med. 2, 293-297.

Malani, A., Trimble, K., Parekh, V., Chenoweth, C., Kaufman, S., and Saint, S. (2007). Review of clinical trials of skin antiseptic agents used to reduce blood culture contamination. Infect. Control. Hosp. Epidemiol. 28, 892-895. doi: $10.1086 / 518456$

Marginson, M. J., Daveson, K. L., and Kennedy, K. J. (2014). Clinical impact of reducing routine blood culture incubation time from 7 to 5 days. Pathology 46, 636-639. doi: 10.1097/PAT.0000000000000167 
Mermel, L. A., Allon, M., Bouza, E., Craven, D. E., Flynn, P., and O'Grady, N. P., et al. (2009). Clinical practice guidelines for the diagnosis and management of intravascular catheter-related infection: 2009 update by the infectious diseases society of America. Clin. Infect. Dis. 49, 1-45. doi: 10.1086/599376

Mermel, L. A., and Maki, D. G. (1993). Detection of bacteremia in adults: consequences of culturing an inadequate volume of blood. Ann. Intern. Med. $119,270-272$.

Mirrett, S., Everts, R. J., and Reller, L. B. (2001a). Controlled comparison of original vented aerobic FAN medium with the new nonvented BacT/ALERT FA medium for culturing blood. J. Clin. Microbiol. 39, 2098-2101. doi: 10.1128/JCM.43.5.2506-2509.2005

Mirrett, S., Petti, C. A., Woods, C. W., Magadia, R., Weinstein, M. P., and Reller, L. B. (2004). Controlled clinical comparison of the BacT/ALERT FN and the Standard Anaerobic SN blood culture medium. J. Clin. Microbiol. 42, 4581-4585. doi: 10.1128/JCM.42.10.4581-4585.2004

Mirrett, S., Weinstein, M. P., Reimer, L. G., Wilson, M. L., and Reller, L. B. (2001b). Relevance of the number of positive bottles in determining clinical significance of coagulase-negative staphylococci in blood cultures. J. Clin. Microbiol. 39, 3279-3281. doi: 10.1128/JCM.39.9.3279-3281.2001

Mitteregger, D., Barousch, W., Nehr, M., Kundi, M., Zeitlinger, M., Makristathis, A., et al. (2013). Neutralization of antimicrobial substances in new BacT/Alert FA and FN Plus blood culture bottles. J. Clin. Microbiol. 51, 1534-1540. doi: 10.1128/JCM.00103-13

Morton, B., Nagaraja, S., Collins, A., Pennington, S. H., and Blakey, J. D. (2015). A retrospective evaluation of critical care blood culture yield - do support services contribute to the "weekend effect"? PLOS ONE 10:e0141361. doi: 10.1371/journal.pone.0141361

Neves, L., Marra, A. R., Camargo, T. Z., dos Santos, M. C., Zulin, F., da Silva, P. C., et al. (2015). Correlation between mass and volume of collected blood with positivity of blood cultures. BMC Res. Notes 8:383. doi: 10.1186/s13104-0151365-8

Novis, D. A., Dale, J. C., Schifman, R. B., Ruby, S. G., and Walsh, M. K. (2001). Solitary blood cultures: a College of American Pathologists Q-probes study of 132,778 blood culture sets in 333 small hospitals. Arch. Pathol. Lab. Med. 125, 1290-1294. doi: 10.1043/0003-9985(2001)125\&lt;1290:SBC\&gt;2.0.CO;2

O'Grady, N. P., Barie, P. S., Bartlett, J. G., Bleck, T., Carroll, K., Kalil, A. C., et al. (2008). Guidelines for evaluation of new fever in critically ill adult patients: 2008 update from the American college of critical care medicine and the infectious disease society of America. Crit. Care Med. 36, 1330-1349. doi: 10.1097/CCM.0b013e318169eda9

Patel, R., Vetter, E. A., Harmsen, W. S., Schleck, C. D., Fadel, H. J., and Cockerill, F. R. 3rd. (2011). Optimized pathogen detection with 30- compared to 20-milliliter blood culture draws. J. Clin. Microbiol. 49, 4047-4051. doi: 10.1128/JCM.01314-11

Petti, C. A., Bhally, H. S., Weinstein, M. P., Joho, K., Wakefield, T., Reller, L. B., et al. (2006). Utility of extended blood culture incubation for isolation of Haemophilus, Actinobacillus, Cardiobacterium, Eikenella, and Kingella organisms: a retrospective multicenter evaluation. J. Clin. Microbiol. 44, 257-259. doi: 10.1128/JCM.44.1.257-259.2006

Pfeiffer, C. D., Samsa, G. P., Schell, W. A., Reller, L. B., Perfect, J. R., and Alexander, B. D. (2011). Quantitation of Candida CFU in initial positive blood cultures. J. Clin. Microbiol. 49, 2879-2883. doi: 10.1128/JCM.00609-11

Pohlman, J. K., Kirkley, B. A., Easley, K. A., Basille, B. A., and Washington, J. A. (1995). Controlled clinical evaluation of BACTEC Plus aerobic/F and BacT/ALERT Aerobic FAN for detection of bloodstream infections. J. Clin. Microbiol. 33, 2856-2858.

Poses, R. M., and Anthony, M. (1991). Availability, wishful thinking, and physicians' diagnostic judgments for patients with suspected bacteremia. Med. Decis. Mak. 11, 159-168.

Public Health England (2014). UK Standards for Microbiology Investigations B 37: Investigation of Blood Cultures (for Organisms other than Mycobacterium Species). Available online at: https://www.gov.uk/government/collections/ standards-for-microbiology-investigations-smi

Reimer, L. G., Wilson, M. L., and Weinstein, M. P. (1997). Update on detection of bacteremia and fungemia. Clin. Microbiol. Rev. 103, 444-465.

Reynes, V. (1947). Densité de l'infection sanguine dans les bactériémies et septicémies. C. R. Seances Soc. Biol. Fil. 141:261.
Riedel, S., Bourbeau, P., Swartz, B., Brecher, S., Carroll, K. C., Stamper, P. D., et al. (2008). Timing of specimen collection for blood cultures from febrile patients with bacteremia. J. Clin. Microbiol. 46, 1381-1385. doi: 10.1128/JCM.02033-07

Ronnberg, C., Mildh, M., Ullberg, M., and Ozenci, V. (2013). Transport time for blood culture bottles: underlying factors and its consequences. Diagn. Microbiol. Infect. Dis. 76, 286-290. doi: 10.1016/j.diagmicrobio.2013.03.031

Roth, A., Wiklund, A. E., Pålsson, A. S., Melander, E. Z., Wullt, M., Cronqvist, J., et al. (2010). Reducing blood culture contamination by a simple informational intervention. J. Clin. Microbiol. 48, 4552-4558. doi: 10.1128/JCM.00877-10

Royer, G., Meusy, A., Bouzinbi, N., Nagot, N., Vande Perre, P., Lamy, B., and colBVH study group. (2015). "Blood culture practices: urgently needed improvement is more easily achieved with the single sampling strategy," in Presented at the 25th European Congress of Clinical and Infectious Diseases (ECCMID), Copenhagen, 25-28.

Salluzzo, R., and Reilly, K. (1991). The rational ordering of blood cultures in the emergency department. Qual. Assur. Util. Rev. 6, 28-31.

Sautter, R. L., Bills, A. R., Lang, D. L., Ruschell, G., Heiter, B. J., and Bourbeau, P. P. (2006). Effects of delayed-entry conditions on the recovery and detection of microorganisms from BacT/ALERT and BACTEC blood culture bottles. J. Clin. Microbiol. 44, 1245-1249. doi: 10.1128/JCM.44.4.1245-1249.2006

Schiffenbauer, J. (2012). Department of Health and Human Services. Supplement approval. 2012. Available Online at: http://www.accessdata.fda.gov/drugsatfda docs/appletter/2012/020832Origs030,021555Orig1s017ltr.pdf (Accessed February 12, 2015).

Schifman, R. B., Bachner, P., and Howanitz, P. J. (1996). Blood culture quality improvement: a college of American pathologists Q-probes study involving 909 institutions and 289572 blood culture sets. Arch. Pathol. Lab. Med. 120, 999-1002.

Schifman, R. B., Strand, C. L., Braun, E., Louis-Charles, A., Spark, R. P., and Fried, M. L. (1991). Solitary blood cultures as a quality assurance indicator. Qual. Assur. Util. Rev. 6, 132-137.

Schifman, R. B., Strand, C. L., Meier, F. A., and Howanitz, P. J. (1998). Blood culture contamination: a college of American pathologists Q-Probes study involving 640 institutions and 497134 specimens from adult patients. Arch. Pathol. Lab Med. 122, 216-221.

Schmitz, R. P. H., Keller, P. M., Baier, M., Hagel, S., Pletz, M. W., and Brunkhorst, F. M. (2013). Quality of blood culture testing - a survey in intensive care units and microbiological laboratories across four European countries. Crit. Care. 17:R248. doi: $10.1186 / \mathrm{cc} 13074$

SFM. (2007). "Hémoculture," in REMIC, Référentiel en Microbiologie Médicale, 3rd Edn., eds G. Cornaglia, R. Courcol, J. L. Hermann, G. Kahlmeter, H. Peigue-Lafeuille, and J. Vila (Paris: Vivactis Plus), 15-20.

Seegmüller, I., Eschenbach, U., Kamereck, K., and Miethke, T. (2004). Sensitivity of the BacT/ALERT FA-medium for detection of Pseudomonas aeruginosa in preincubated blood cultures and its temperature-dependence. J. Med. Microbiol. 53, 869-874. doi: 10.1099/jmm.0.45533-0

Shapiro, N. I., Wolfe, R. E., Wright, S. B., Moore, R., and Bates, D. W. (2008). Who needs a blood culture? A prospectively derived and validated prediction rule. J. Emerg. Med. 35, 255-264. doi: 10.1016/j.jemermed.2008.04.001

Snyder, S. R., Favoretto, A. M., Baetz, R. A., Derzon, J. H., Madison, B. M., Mass, D., et al. (2012). Effectiveness of practices to reduce blood culture contamination: a laboratory medicine best practices systematic review and meta-analysis. Clin. Biochem. 45, 999-1011. doi: 10.1016/j.clinbiochem.2012. 06.007

Souvenir, D., Anderson, D. E. Jr., Palpant, S., Mroch, H., Askin, S., Anderson, J., et al. (1998). Blood cultures positive for coagulase-negative staphylococci: antisepsis, pseudobacteremia, and therapy of patients. J. Clin. Microbiol. 36, 1923-1926.

van Ingen, J., Hilt, N., and Bosboom, R. (2013). Education of phlebotomy teams improves blood volume in blood culture bottles. J. Clin. Microbiol. 51, 1020-1021. doi: 10.1128/JCM.03068-12

Vitrat-Hincky, V., Francois, P., Labarere, J., Recule, C., Stahl, J. P., and Pavese, P. (2011). Appropriateness of blood culture testing parameters in routine practice. Results from a cross-sectional study. Eur. J. Clin. Microbiol. Infect. Dis. 30, 533-539. doi: 10.1007/s10096-010-1115-8

Wain, J., Diep, T. S., Ho, V. A., Walsh, A. M., Nguyen, T. T., Parry, C. M., et al. (1998). Quantitation of bacteria in blood of typhoid fever patients 
and relationship between counts and clinical features, transmissibility, and antibiotic resistance. J. Clin. Microbiol. 36, 1683-1687.

Warren, M., and Herrick, W. W. (1916). Analysis of one hundred and thirty four cases of bacteremia. Am. J. Med. Sci. 151, 556-577.

Washer, L. L., Chenoweth, C., Kim, H. W., Rogers, M. A., Malani, A. N., Riddell, J. IV., et al. (2013). Blood culture contamination: a randomized trial evaluating the comparative effectiveness of 3 skin antiseptic interventions. Infect. Control. Hosp. Epidemiol. 34, 15-21. doi: 10.1086/668777

Washington, J. A (1975). Blood cultures: principles and techniques. Mayo Clin. Proc. 52, 91-98.

Washington, J. A. (1992). An international multicenter study of blood culture practices: the international collaborative blood culture study group. Eur. J. Clin. Microbiol. Infect. Dis. 11, 1115-1128.

Watson, K. C., and Pietermaritzburg, C. B. (1955). Isolation of Salmonella typhi from the blood stream. J. Lab. Clin. Med. 46, 128-134.

Weinstein, M. P. (1996). Current blood culture methods and systems: clinical concepts, technology, and interpretation of results. Clin. Infect. Dis. 23, 40-44.

Weinstein, M. P., Mirrett, S., Reimer, L. G., Wilson, M. L., Smith-Elekes, S., Chuard, C. R., et al. (1995). Controlled evaluation of BacT/ALERT standard aerobic and FAN aerobic blood culture bottles for detection of bacteremia and fungemia. $J$. Clin. Microbiol. 33, $978-981$.

Werner, A. S., Cobbs, C. G., Kaye, D., and Hook, E. W. (1967). Studies on the bacteremia of bacterial endocarditis. JAMA 202, 199-203.

Willems, E., Smismans, A., Cartuyvels, R., Coppens, G., Van Vaerenbergh, K., Van den Abeele, et al. (2012). The preanalytical optimization of blood cultures: a review and the clinical importance of benchmarking in 5 Belgian hospitals. Diagn. Microbiol. Infect. Dis. 73, 1-8. doi: 10.1016/j.diagmicrobio.2012. 01.009

Wilson, M. L., Mirrett, S., Meredith, F. T., Weinstein, M. P., Scotto, V., and Reller, L. B. (2001). Controlled clinical comparison of BACTEC Plus Anaerobic/F to standard Anaerobic/F as the anaerobic companion bottle to plus Aerobic/F medium for culturing blood from adults. J. Clin. Microbiol. 39, 983-989. doi: 10.1128/JCM.39.3.983-989.2001

Wilson, M. L., Mirrett, S., Reller, L. B., Weinstein, M. P., and Reimer, L. G. (1993). Recovery of clinically important microorganisms from the BacT/Alert blood culture system does not require testing for seven days. Diagn. Microbiol. Infect. Dis. 16, 31-34.

Wilson, M. L., Mitchell, M., Morris, A. J., Murray, P. R., Reimer, R. G., Reller, L. B., et al. (2007). Principles and Procedures for Blood Cultures; Approved Guidelines, CLSI Document M47-A, 27. Wayne, PA: Clinical and Laboratory Standard Institute.

Wilson, M. L., Weinstein, M. P., Mirrett, S., Reimer, L. G., Feldman, R. J., Chuard, C. R., et al. (1995). Controlled evaluation of BacT/alert standard anaerobic and FAN anaerobic blood culture bottles for the detection of bacteremia and fungemia. J. Clin. Microbiol. 33, 2265-2270.

Wilson, M. L., Weinstein, M. P., and Reller, L. B. (1994). Automated blood culture systems. Clin. Lab. Med. 14,149-169.

Zwang, O., and Albert, R. K. (2006). Analysis of strategies to improve cost effectiveness of blood cultures. J. Hosp. Med. 1, 272-276. doi: 10.1002/jhm.115

Conflict of Interest Statement: The authors declare that the research was conducted in the absence of any commercial or financial relationships that could be construed as a potential conflict of interest.

Copyright (c) 2016 Lamy, Dargère, Arendrup, Parienti and Tattevin. This is an openaccess article distributed under the terms of the Creative Commons Attribution License (CC BY). The use, distribution or reproduction in other forums is permitted, provided the original author(s) or licensor are credited and that the original publication in this journal is cited, in accordance with accepted academic practice. No use, distribution or reproduction is permitted which does not comply with these terms. 